\title{
EFICIÊNCIA DE INSETICIDAS NO CONTROLE DE Sitophilus oryzae (L.) (COLEOPTERA: CURCULONIDAE) E Rhyzopertha dominica (FAB.) (COLEOPTERA: BOSTRICHIDAE) EM CEVADA ARMAZENADA.
}

\author{
Efficiency os Insecticides in the Control of Sitophilus oryzae (L) \\ (Coleoptera: Curculionidae) e Rhyzopertha dominica (fab.) \\ (Coleoptera: Bostrichidae) in Stored Barley.
}

\author{
Paulo R. V. da S. Pereira ${ }^{1}$ \\ Rui S. Furiatti ${ }^{2}$
}

\section{Resumo}

Com o propósito de testar inseticidas no controle de Sitophilus oryzae e Rhyzopertha dominica, em grãos de cevada, foi realizado um experimento no laboratório de fitotecnia da Universidade Estadual de Ponta Grossa, PR. Os tratamentos foram feitos utilizando-se um pulverizador propulsionado a ar, equipado com bico pneumático. Em todos os tratamentos utilizou-se 0,005 l de água. $\mathrm{kg}^{-1}$ de cevada, acondicionado em sacos plásticos. Na testemunha apenas água foi aplicada com o mesmo aparelho e na quantidade citada. Para cada tratamento utilizou-se $1,2 \mathrm{~kg}$ de cevada, a qual foi armazenada em saco de ráfia e mantida em sala climatizada. A cada 15 dias, $50 \mathrm{~g}$ desta cevada foi infestada com 10 indivíduos dos insetos supracitados. A avaliação dos tratamentos foi feita 15 dias após a infestação. Desta forma, foram feitas avaliações aos 30, 60 e 90 dias após o tratamento. No controle de S.oryzae, bifentrin (dose de $16 \mathrm{ml}^{-\mathrm{t}^{-1}}$ ) mostrou-se ineficiente em todas as avaliações (30, 60 e 90 dias após a aplicação). Deltametrina + BPO (15 ml.t $\left.{ }^{-1}\right)$ mostrou-se eficiente até os 30 dias, perdendo gradativamente a eficiência a partir dos 60 dias após a aplicação. Bifentrin (dose de 32 ml.t $\left.{ }^{-1}\right)$, fenitrothion $\left(10 \mathrm{ml}^{\left.-\mathrm{t}^{-1}\right)}\right.$ e pirimiphos methyl $\left(10 \mathrm{ml}^{\left.-\mathrm{t}^{-1}\right)}\right.$ mostraram-se eficientes até o término do experimento aos 90 dias após a aplicação. No controle de R. dominica, somente bifentrin (doses de 16 e 32 $\mathrm{ml.t}^{-1}$ ) foi eficiente até $\mathrm{o}$ término do experimento aos 90 dias após a aplicação. Os demais ingredientes ativos testados (pirimiphos methyl, Deltametrina + BPO e fenitrothion) mostraram-se ineficientes no controle deste inseto em todas as avaliações.

Palavras-chave: Controle químico; Pragas de produtos armazenados; Cevada.

1 Pesquisador Dr. lotado na Embrapa Roraima, BR 174, km 8, Distrito Industrial, Boa Vista, Caixa postal 133, 69301-970, RR, e-mail: paulo@cpafrr.embrapa.br

2 Prof. Dr. lotado no Departamento de Fitotecnia e Fitossanidade, UEPG, Praça Santos Andrade, s/n, 84010-330, Ponta Grossa, PR, e-mail: furiatti@centerline.com.br 


\section{Abstract}

The efficiency of insecticides was determined against S. oryzae (L) (Coleoptera: Curculionidae) and Rhyzopertha dominica (Fab.) (Coleoptera: Bostrichidae) exposed to treated barley. The treatments were applied with a propelling air atomizer using $50 \mathrm{ml}$ of mixture. $\mathrm{kg}^{-1}$ of barley except in the control, when was used only water. For each treatment $1.2 \mathrm{~kg}$ of barley were treated in plastic bags and stored in raffia bags in a climatized room $\left(25 \pm 2^{\circ} \mathrm{C}\right)$. Fifteen days after treatment $200 \mathrm{~g}$ of the treated barley from each treatment were infested with 40 adult insects of each species, and this procedure was repeated every 30 days until 90 days after the treatment. The number of dead insects was counted 15 days after each infestation, and the evaluations were made at 30, 60, 90 days after the insecticide application. In the control of S. oryzae, bifenthrin (16 ml.t ${ }^{-1}$ ) was inefficient in all evaluations (30,60 and 90 days after insecticide treatments). Deltamethrin + piperonyl butoxide $\left(15 \mathrm{ml}^{-\mathrm{t}^{-1}}\right)$ was efficient only until 30 days after treatment. Bifenthrin $\left(32 \mathrm{ml} . \mathrm{t}^{-1}\right)$, fenitrothion $\left(10 \mathrm{ml}^{-\mathrm{t}^{-1}}\right)$ and pirimiphos methyl $\left(10 \mathrm{ml.t}{ }^{-1}\right)$ were efficient until the end of the experiment, at 90 days after insecticide treatments. In the control of R. Dominica, only bifenthrin (16 and $32 \mathrm{ml}^{\left.-\mathrm{t}^{-1}\right) \text { was }}$ efficient until the end of the experiment, 90 days after insecticide treatments. The other active ingredients tested (fenitrothion, deltamethrin + piperonyl butoxide and pirimiphos methyl) were inefficient during all evaluations (30, 60 and 90 days).

Keywords: Chemical control; stored product pests; barley

\section{Introdução}

A FAO estima que as perdas quantitativas anuais causadas por pragas durante o período de armazenamento de grãos são da ordem de $10 \%$ da produção mundial. Esta estimativa refere-se, apenas, ao ataque dos insetos ao embrião e ao endosperma das sementes, não entrando em consideração o aquecimento da massa de grãos provocada pela atividade dos insetos, conseqüente ataque de fungos e também a diminuição do valor nutritivo dos grãos (ALMEIDA, 1989).

No Brasil, em função das precárias condições de armazenamento no meio rural e de condições climáticas favoráveis ao crescimento da população de pragas, as perdas atingem cerca de $10 \%$ da produção total de grãos (VENDRAMIN et al., 1985). A baixa disponibilidade de inseticidas registrados para o controle de pragas de produtos armazenados contribui para o agravamento deste problema, uma vez que nem todos os produtos podem ser utilizados para todas as pragas e nas diversas situações de armazenamento encontradas no Brasil (LORINI; SCHNEIDER, 1994).

Na desinfestação prévia dos focos residuais de pragas em armazéns, paióis, tulhas, silos e graneleiros, recomenda-se a pulverização dos pisos, paredes e outras partes acessíveis. O teor de umidade dos grãos a serem armazenados não deve exceder a determinados limites (ao redor de 12 a $13 \%$ para cereais e de 11 a $12 \%$ para soja e feijão).
No Brasil, Pacheco et al. (1988) encontraram linhagens de S. oryzae insensíveis ao inseticida deltamethrina, mesmo com uma dose de 2 mg.kg ${ }^{-1}$ de trigo.

Yadav (1988), trabalhando com trigo armazenado em saco de juta, demonstrou que a toxicidade da deltamethrina para S. oryzae e R. dominica persistiu por 120 a 170 dias na superfície da juta, e a proteção contra esses insetos durou por 6 meses de armazenagem.

Schiffers et al. (1989) constataram que a deltamethrina foi o inseticida mais eficaz no controle de $\mathrm{R}$. dominica e S. oryzae em trigo.

Bareth; Gupta (1989a), trabalhando com $\mathrm{R}$ dominica em trigo, mostraram que a deltamethrina plus a 0,015\% e deltamethrina a 0,075 ppm foram os produtos mais eficientes no controle deste inseto.

Bareth; Gupta (1989b) testaram 6 inseticidas no controle de R. dominica, concluindo que a deltamethrina a $2 \mathrm{ppm}$ mostrou a melhor eficácia contra esse inseto, seguido por fenitrothion a $20 \mathrm{ppm}$. Demonstraram também que nenhum dos inseticidas alterou a germinação das sementes.

Suchita et al. (1989), baseando-se na CL50 do S. oryzae, concluíram que o fenpropathrim foi o produto que mostrou o menor controle, quando comparado com cipermethrin, malathion, fenvalerate e flucitrinate.

Islam et al. (1989), comparando deltamethrina com carbaril contra S. oryzae, concluíram que a deltamethrina foi mais efetiva por um período de nove meses em trigo armazenado. 
Giga; Zvoutete (1990), trabalhando com milho infestado com S. zeamais, observaram que 0 fenitrothion e a deltamethrina foram eficientes até 120 e 90 dias após o tratamento, respectivamente.

Duguet; Quan (1990) demonstraram que a deltamethrina $(0,5 \mathrm{ppm}+5,0 \mathrm{ppm}$ de butóxido de piperonila, BPO) protegeu o arroz contra $R$. dominica, S. zeamais e S. oryzae em trabalho realizado na China.

A aplicação de deltamethrina a $1 \mathrm{mg} \mathrm{kg}^{-1}$, com BPO a $8 \mathrm{mg} \mathrm{kg}^{-1}$, controlou as progênies de

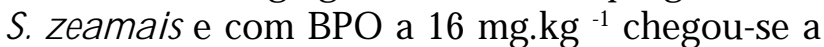
48 semanas de proteção. Entretanto papéis impregnados com deltamethrina sinergizada foram menos efetivos contra biótipos de S. zeamais resistentes a deltamethrina e organofosforados (SAMSON et al., 1990).

Guedes; Silva (1992) obtiveram bom controle de S. zeamais com a deltamethrina $(0,43 \mathrm{ppm})$ em milho armazenado, após 30, 60, 90, 120, 150 e 180 dias do tratamento.

sEste trabalho teve por objetivo avaliar a eficácia de inseticidas no controle de Sitophilus oryzae (L.) (Coleoptera: Curculionidae) e Rhyzopertha dominica (Fab.) (Coleoptera: Bostrichidae) em cevada armazenada, na região de Curitiba, PR.

\section{Material e métodos}

O experimento foi realizado no laboratório de fitotecnia da Universidade Estadual de Ponta Grossa, $\mathrm{PR}$, onde foram mantidas em sala climatizada $\left(25 \pm 2^{\circ} \mathrm{C}\right)$ as colônias de $\mathrm{S}$. oryzae e de $\mathrm{R}$. dominica, provenientes de diversos pontos do Estado de Santa Catarina. O delineamento estatístico utilizado foi de blocos casualizados, com quatro repetições.

Cada parcela foi constituída de 1,2 kg de cevada, que após tratadas foram acondicionados em sacos de ráfia e armazenados em sala climatizada $\left(25 \pm 2^{\circ} \mathrm{C}\right)$.

A aplicação dos inseticidas foi efetuada com um pulverizador manual propulsionado a ar, equipado com bico pneumático com vazão de 5,0 l.t ${ }^{-1}$. Cada parcela recebeu $5 \mathrm{ml}$ de calda por $\mathrm{kg}$ de grãos de cevada e na testemunha apenas água. Os inseticidas foram aplicados dentro de sacos plásticos de $0,4 \times 0,5 \mathrm{~m}$ e homogeneizados por dois minutos.

Nos tratamentos utilizou-se: o inseticida piretróide, bifenthrin (Starion na dosagem de $16 \mathrm{e}$ $32 \mathrm{ml}^{\mathrm{t}}{ }^{1}$ ), o organofosforado, fenitrothion (Sumigran ${ }^{\circledR} 500 \mathrm{CE}$, na dosagem de $10 \mathrm{ml}^{-1} \mathrm{t}^{1}$ ); o piretróide, deltametrina ( $\mathrm{k}-\mathrm{Obiol}{ }^{\circledR}$ 2,5 CE na dosagem de $15 \mathrm{ml}^{\mathrm{t}} \mathrm{t}^{ }$), o organofosforado, pirimiphos methyl (Actellic $500 \mathrm{CE}$ na dosagem de $10 \mathrm{ml} . \mathrm{t}^{1}$ ).

Para a avaliação da eficiência, em intervalos de 15 dias após a aplicação dos inseticidas, $50 \mathrm{~g}$ de cada parcela foram infestadas com 10 insetos adultos, não sexados e sem padronização de idade das espécies testadas, acondicionadas em copos plásticos de 0,5 l em sala climatizada $\left(25 \pm 2{ }^{\circ} \mathrm{C}\right)$. A avaliação do número de insetos mortos foi feita 15 dias após cada infestação, ou seja, aos 30, 60 e 90 dias após a aplicação dos inseticidas.

Considerou-se eficiente o inseticida que não apresentou diferença estatística a $100 \%$ de mortalidade de S. oryzae e R. dominica, e diferença estatística significativa da testemunha.

O número de insetos mortos por parcela foi submetido à análise de variância e as diferenças entre as médias discriminadas pelo teste de Tukey a 5\%. A eficiência relativa dos inseticidas foi calculada pela fórmula de Abbot (ABBOTT, 1925).

\section{Resultados e discussão}

No controle de Sitophilus oryzae, bifentrin (dose de $16 \mathrm{ml}^{\mathrm{t}} \mathrm{t}^{ }$) mostrou-se ineficiente em todas as avaliações (30, 60 e 90 dias após a aplicação). Deltametrina + BPO (15 ml.t $\left.{ }^{1}\right)$ mostrou-se eficiente até os 30 dias, perdendo gradativamente a eficiência a partir dos 60 dias após a aplicação. Bifentrin (dose de $32 \mathrm{ml}^{-1}$ ), fenitrothion (10 ml. $\mathrm{t}^{1}$ ) e pirimiphos methyl (10 ml. $\left.\mathrm{t}^{1}\right)$ mostraram-se eficientes até o término do experimento, aos 90 dias após a aplicação (TABELA 1). 


\section{TABELA 1 - Número médio de indivíduos mortos $(\mathrm{X})$ de Sitophilus oryzae e porcentagem de} eficácia dos inseticidas (E\%) aos 30, 60 e 90 dias após o tratamento dos grảos de cevada. Table 1 - Average number of dead individuals (x) of Sitophilus oryzae and percentage of effectiveness of the insecticides ( $\mathrm{E} \%$ ) at 30, 60 and 90 days after the treatment of the barley grains.

\begin{tabular}{|c|c|c|c|c|c|c|c|}
\hline \multirow{3}{*}{ Tratamentos $^{1}$} & \multirow[b]{3}{*}{ Dosagem $\left(\mathrm{ml} \cdot \mathrm{t}^{-1}\right)$} & \multicolumn{6}{|c|}{ Dias a pós o tratamento } \\
\hline & & \multicolumn{2}{|c|}{30} & \multicolumn{2}{|c|}{60} & \multicolumn{2}{|c|}{90} \\
\hline & & $\mathrm{X}$ & $\mathrm{E} \%$ & $\mathrm{X}$ & $\mathrm{E} \%$ & $\mathrm{X}$ & $\mathrm{E} \%$ \\
\hline 1 & 16 & $7,75 \mathrm{~b}$ & 76,92 & $8,50 \mathrm{~b}$ & 82,85 & $6,75 \mathrm{c}$ & 63,88 \\
\hline 2 & 32 & $10,0 \mathrm{a}$ & 100,0 & $10,0 \mathrm{a}$ & 100,0 & $10,0 \mathrm{a}$ & 100,0 \\
\hline 3 & 10 & $10,0 \mathrm{a}$ & 100,0 & $10,0 \mathrm{a}$ & 100,0 & $10,0 \mathrm{a}$ & 100,0 \\
\hline 4 & 15 & $10,0 \mathrm{a}$ & 100,0 & $9,75 \mathrm{ab}$ & 97,14 & $8,75 \mathrm{~b}$ & 86,11 \\
\hline 5 & 10 & $10,0 \mathrm{a}$ & 100,0 & $10,0 \mathrm{a}$ & 100,0 & $10,0 \mathrm{a}$ & 100,0 \\
\hline 6 & - & $0,25 \mathrm{c}$ & - & $1,25 \mathrm{c}$ & - & $1,0 \mathrm{~d}$ & - \\
\hline \multicolumn{8}{|l|}{$\mathrm{CV} \%$} \\
\hline F & & & & & & & \\
\hline
\end{tabular}

\footnotetext{
${ }^{1}$ Tratamentos: 1 = bifenthrin (dos.1); 2 = bifenthrin (dos.2); 3 =fenitrothion; $4=$ deltametrina + BPO; $5=$ pirimiphos methyl; $6=$ testemunha .
}

No controle de Rhyzopertha dominica, somente bifentrin (doses de 16 e $32 \mathrm{ml} . \mathrm{t}^{1}$ ) foi eficiente até o término do experimento, aos 90 dias após a aplicação. Os demais ingredientes ativos testados (pirimiphos methyl, Deltametrina + BPO e fenitrothion) mostraram-se ineficientes no controle deste inseto em todas as avaliações, aos 30, 60 e 90 dias após a aplicação (TABELA 2).

\section{TABELA 2 - Número médio de indivíduos mortos (X) de Rhyzopertha dominica e porcentagem} de eficácia dos inseticidas (E\%) aos 30, 60 e 90 dias após o tratamento dos grãos de cevada.

Table 2 - Average number of dead individuals (x) of Rhyzopertha dominica and percentage of effectiveness of the insecticides ( $\mathrm{E} \%$ ), at 30, 60 and 90 days after the treatment of the barley grains.

\begin{tabular}{|c|c|c|c|c|c|c|c|}
\hline \multirow{3}{*}{ Tratamentos $^{1}$} & \multirow[b]{3}{*}{ Dosagem $\left(\mathrm{ml} \cdot \mathrm{t}^{-1}\right)$} & \multicolumn{6}{|c|}{ Dias a pós o tratamento } \\
\hline & & \multicolumn{2}{|c|}{30} & \multicolumn{2}{|c|}{60} & \multicolumn{2}{|c|}{90} \\
\hline & & $\mathrm{x}$ & $\mathrm{E} \%$ & $\mathrm{x}$ & $\mathrm{E} \%$ & $\mathrm{X}$ & $\mathrm{E} \%$ \\
\hline 1 & 16 & 10,0 & 100,0 & 10,0 & 100,0 & 10,0 & 100,0 \\
\hline 2 & 32 & 10,0 & 100,0 & 10,0 & 100,0 & 10,0 & 100,0 \\
\hline 3 & 10 & 1,75 & 5,71 & 0,75 & 1,0 & 2,75 & 17,14 \\
\hline 4 & 15 & 5,75 & 51,42 & 6,75 & 64,86 & 6,25 & 57,14 \\
\hline 5 & 10 & 4,50 & 37,14 & 4,50 & 40,54 & 5,0 & 42,85 \\
\hline 6 & - & 1,25 & - & 0,75 & - & 1,25 & - \\
\hline \multicolumn{8}{|l|}{$\mathrm{CV} \%$} \\
\hline F & & & & & & & \\
\hline
\end{tabular}

\footnotetext{
${ }^{1}$ Tratamentos: 1 = bifenthrin (dos.1); $2=$ bifenthrin (dos. 2$) ; 3=$ fenitrothion; $4=$ deltametrina + BPO; $5=$ pirimiphos methyl; $6=$ testemunha .
} 
Para o bifentrin, os resultados obtidos no controle de S. oryzae e R. dominica estão semelhantes aos obtidos por Schiffers et al. (1989), que observaram a eficiência deste ingrediente ativo contra ambos insetos. Estes pesquisadores indicam ainda a R. dominica como mais suscetível que S. oryzae, fato este que também pode ser observado neste trabalho, com as duas doses de bifentrin (16 e $32 \mathrm{ml} . \mathrm{t}^{1}$ ), sendo eficientes contra R. dominica e somente a dose de $32 \mathrm{ml}^{-1}$ eficiente contra $\mathrm{S}$. oryzae.

Para o fenitrothion, os resultados obtidos no controle de S. oryzae são concordantes com os obtidos por Pereira et al. (1997); Funiatti et al. (1999) quando foi observada eficiência deste ingrediente ativo até 180 dias após a aplicação em grãos de milho e trigo, respectivamente. No entanto, em arroz em casca armazenado, Pinto Jr. et al. (1997) observaram que o fenitrothion foi eficiente apenas até os 30 dias, em um acompanhamento até os 180 dias após a aplicação. Os resultados obtidos no controle de $\mathrm{R}$ dominica são concordantes com os obtidos por Pereira et al. (1997); Pinto Jr. et al. (1997); Furiatti et al. (1999), que observaram a ineficiência de fenitrothion em todas as avaliações em um acompanhamento até os 180 dias após a aplicação em grãos de milho, arroz em casca e trigo, respectivamente. Entretanto Bareth; Gupta (1989a), testando inseticidas em grãos de trigo, registraram a eficiência de fenitrothion contra R. dominiica.

Para a deltametrina $+\mathrm{BPO}$, os resultados obtidos no controle de S. oryzae são concordantes com os observados por Bitran et al. (1991), Pereira et al. (1997); Pinto Jr. et al. (1997) que, testando inseticidas na proteção de milho, trigo e arroz em casca, observaram um decréscimo na ação residual deste ingrediente ativo. Furiatti et al. (1999), entretanto, observaram a sua eficiência com S. oryzae até 150 dias após a aplicação. Os resultados obtidos no controle de $\mathrm{R}$. dominica estão discordantes com os obtidos por Pereira et al. (1997); Pinto Jr. et al. (1997); Furiatti et al. (1999) que observaram a eficiência de deltametrina + BPO até os 120 dias após a aplicação em grãos de milho, arroz em casca e trigo, respectivamente. Para Bitran et al. (1991), a deltametrina, em grãos de trigo, foi o melhor tratamento contra R. dominica.

Para o pirimiphos methyl, os resultados obtidos no controle de S. oryzae e R. dominica são concordantes com Bitran et al. (1991); Collins; Cook (1998), que observaram a longa ação residu- al deste ingrediente ativo no controle de S. oryzae e sua ineficiência no controle de R dominica.

Pelos resultados obtidos neste trabalho e confirmados por Pereira et al. (1997); Furiatti et al. (1999), observou-se, para um mesmo ingrediente ativo com uma mesma dose, uma grande diferença em sua eficiência quando se mudou a pragaalvo. Desta maneira, pode-se concluir que as informações aqui obtidas são de muita importância prática na escolha do inseticida a ser usado no controle dos insetos em questão, uma vez que estes, muitas vezes, são encontrados associados infestando a massa de grãos. Além disso, fica caracterizada a importância da identificação correta da praga infestante para a escolha correta do ingrediente ativo a ser usado e o conseqüente sucesso da prática de controle.

\section{Referências}

ABOTT, W. S. A method of computing the effectiveness of an insecticide. J. Econ. Entomol. v. 18, p. 265 - 267, 1925.

ALMEIDA, A. A. Natureza dos danos causados por insetos em grãos armazenados. In: CONGRESSO BRASILEIRO DE ENTOMOLOGIA, 11., Campinas, 1989. Anais... Campinas, 1989. v. 4, p. 16 - 32.

BARETH, S. S.; GUPTA, H. C. Efficacy of gunny bag and seed treatment on the natural infestation of Rhyzopertha dominica on stored wheat. Seed Res. v. 17, n. 2, p. 178-181, 1989a.

BARETH, S. S.; GUPTA, H. C. Efficacy of six insectides for the protection of stored wheat seeds against Rhyzoperta dominica (Fab.) Seed Res. v. 17, n. 1, p. 43-46, 1989 b.

BITRAN, E. A.; CAMPOS, T. B.; SUPLCY FILHO, N.; CHIBA, S. Avaliação da ação residual de alguns inseticidas na proteção de grão de milho, trigo e arroz contra pragas de armazenamento. Arq. Inst. Biol. v. 58, n. 1-2, p. 43-50, 1991.

COLUNS, D. A.; COOK D. A. Periods of protection provided by different formulations of pirimiphos methyl and etrimfos, when admixed with wheat, against four susceptible storage beetle pests. CropProtection. v. 17, n. 6, p. 521- 528, 1998.

DUGUET, J.; QUAN, L Evaluation of the effectivness of deltamethrin spray or dust on rice husks against 
stored products pests on stored rice in southern of China. Agronomie Tropicale, v. 45, n. 2, p. 107113, 1990.

FURIATTI, R. S.; PEREIRA, P. R. V. DA S.; PINTO JUNIOR, A. R; LAZZARI, F. A. Avaliação de inseticidas no controle de Sitophilus oryzae (L.) (Coleoptera: Curculionidae) e Rhyzopertha dominica (Fab.) (Coleoptera: Bostrichidae) em trigo. Revista Brasileira de Zoologia, v. 16, n. 1, p. 221-226, 1999.

GIGA, D. P.; ZVOUTETE, P. The evaluation of different insecticides for the protection of maize against some stored product pests. I. Pest Cont. v. 32, n. 1, p. 10-13, 1990.

GUEDES, R. N. C.; SILVA, F. A. P. DA Avaliação do efeito residual do inseticida abamectin no controle de Sitophilus zeamais Motschulsky (COLEOPTERA: CURCULIONIDAE) em milho armazenado. Rev. Ceres, v. 39, n. 225, p. 435-442, 1992.

ISLAM, N.; BHUIYAH, M. I. M.; BEGUM, A.; KARIM, M. A. Comparative efficacy of different material against Sitophilu s oryzae L infesting maize seeds in storage. Bang. J. of Zool. v. 17, n. 2, p. 175-178, 1989.

LORINI, I.; S. SCHNEIDER. Pragas de Grãos Armazenados: resultados de pesquisa. Passo Fundo, Embrapa - CNPT. 1994. 48 p.

PACHECO, I. A.; SARTORI, M. R.; YOKOMIZO, Y. Suscetibilidade de Sitophilusoryzae(COLEOPTERA: CURCULIONIDAE) ao piretróide deltametrina. Coletânea do Instituto de Tecnologia de Alimentos, v. 18, n. 1, p. 84-94, 1988.

PEREIRA, P. R. V. S. et al. Avaliação de inseticidas no controle de Sitophilus oryzae (L) (Coleoptera: Bostrichidae) e Rhyzopertha dominica (Fab.) (Coleoptera: Bostrichidae) em milho armazenado. In: CONGRESSO BRASILEIRO DEENTOMOLOGIA, 17., Salvador, 1997. Anais... Salvador: Sociedade Entomológica do Brasil, 1997.v. 26, n. 3, p. 411-416.
PINTO JUNIOR, A. R.; FURIATTI, R. S.; PEREIRA, P. R. V. S.; LAZZARI, F. A. Avaliação de inseticidas no controle de Sitophilus oryzae (L.) (Coleoptera: Bostrichidae) e Rhyzopertha dominica (Fab.) (Coleoptera: Bostrichidae) em arroz armazenado. In: CONGRESSO BRASILEIRO DEENTOMOLOGIA, 17., Salvador, 1997. Anais... Salvador: Sociedade Entomológica do Brasil, 1997.v. 26, n. 2, p. 285-290.

SAMSO N, P. R; PARKER, R. J.; HAL, E. A. Synergized deltamethrin as a protectant against Sitophilus zeamais Motsch. and S. oryzae (L.)(COLEOPTERA: CURCULIONIDAE) on stored maize. J. Stored Prod. Res. v. 26, n. 3, p. 155-161, 1990.

SCHIFFERS, B. C., et al. The comparative toxicity of five pyrethroid inseticides to six insect pests of stored products. Medelingen van de Faculteit Landbouwwetenschappen, Rijksuniversiteit Gent, v. 54, n. 3b, p. 1095- 1103, 1989.

SUCHITA, M. G.; REDDY, G. P. V.; MURTHY, M. M. $\mathrm{K}$. Relative efficacy of pyretroids againstrice weevil (Sitophilus oryzae L) infesting stored wheat. I. J. of Plant Prot., v. 17, n. 2, p. 243-246, 1989.

VENDRAMIN, J. D.; FERNANDES, L C.; REIS, FILHO, W. Biologia comparada de Erinnyis ello ello (L,1758) (Lepdoptera, Sphingidae) em mandioca. Poliagro, v. 7, n. 2, p. 11-13, 1985.

YADAV, T. D. Efficacy of deltamethrin against storage insects in rice and wheat under FCI's storage system. Pest. v. 22, n. 12, p. 39-43, 1988. 